\section{(A) Check for updates}

Cite this: Analyst, 2019, 144, 3056

\title{
Screening of two-photon activated photodynamic therapy sensitizers using a 3D osteosarcoma model $\uparrow$
}

\author{
Agnes Dobos, ${ }^{\mathrm{a}, \mathrm{b}}$ Wolfgang Steiger, ${ }^{\mathrm{a}, \mathrm{b}}$ Dominik Theiner, ${ }^{a}$ Peter Gruber, ${ }^{\mathrm{a}, \mathrm{b}}$ \\ Markus Lunzer, (D) a,b,c Jasper Van Hoorick, (D) d,e Sandra Van Vlierberghe (D) d,e and \\ Aleksandr Ovsianikov (D) *a,b
}

Photodynamic therapy (PDT) involves a photosensitizing agent activated with light to induce cell death. Two-photon excited PDT (TPE-PDT) offers numerous benefits compared to traditional one-photon induced PDT, including an increased penetration depth and precision. However, the in vitro profiling and comparison of two-photon photosensitizers (PS) are still troublesome. Herein, we report the development of an in vitro screening platform of TPE-PS using a 3D osteosarcoma cell culture. The platform was tested using three different two-photon (2P) active compounds - a 2P sensitizer P2CK, a fluorescent dye Eosin $\mathrm{Y}$, and a porphyrin derivative (TPP). Their 2P absorption cross-sections $\left(\sigma_{2 \mathrm{PA}}\right)$ were characterised using a fully automated $z$-scan setup. TPP exhibited a remarkably high $\sigma_{2 \mathrm{PA}}$ at $720 \mathrm{~nm}$ (8865 GM) and P2CK presented a high absorption at $850 \mathrm{~nm}$ (405 GM), while Eosin Y had the lowest 2P absorption at the studied wavelengths ( $<100 \mathrm{GM})$. The cellular uptake of PS visualized using confocal laser scanning microscopy showed that both TPP and P2CK were internalized by the cells, while Eosin Y stayed mainly in the surrounding media. The efficiency of the former two TPE-PS was quantified using the PrestoBlue metabolic assay, showing a significant reduction in cell viability after two-photon irradiation. The possibility of damage localization was demonstrated using a co-culture of adipose derived stem cells together with osteosarcoma spheroids showing no signs of damage to the surrounding healthy cells after TPE-PDT.

\author{
Received 11th January 2019 \\ Accepted 6th March 2019 \\ DOI: 10.1039/c9an00068b \\ rsc.li/analyst
}

\section{Introduction}

Photodynamic therapy (PDT) typically comprises three individually harmless components: a photosensitizing agent (PS), light and oxygen to induce cell damage. PDT has been extensively researched for multiple clinical applications such as the treatment of cancer and several other diseases, including a therapy for posterior capsule opacification and age-related macular degeneration. ${ }^{1,2}$ PDT could also provide possible

\footnotetext{
${ }^{a}$ TU Wien, Institute of Materials Science and Technology, Getreidemarkt 9, 1060 Vienna, Austria. E-mail: Aleksandr.Ovsianikov@tuwien.ac.at

${ }^{b}$ Austrian Cluster for Tissue Regeneration, Austria. http://www.tissue-regeneration.at ${ }^{c}$ TU Wien, Institute of Applied Synthetic Chemistry, Getreidemarkt 9, 1060 Vienna, Austria

${ }^{d}$ Ghent University, Polymer Chemistry and Biomaterials Group, Centre of Macromolecular Chemistry, Krijgslaan 281, S4, 9000 Ghent, Belgium

${ }^{e}$ Brussels Photonics, Department of Applied Physics and Photonics, Vrije Universiteit Brussel and Flanders Make, Pleinlaan 2, 1000 Brussels, Belgium

$\dagger$ Electronic supplementary information (ESI) available. See DOI: 10.1039/ c9an00068b
}

alternatives to eliminate pathogenic microorganisms, such as viruses, fungi, yeast and antibiotic resistant bacteria. ${ }^{3}$

In PDT, energy is transferred from light to oxygen by the PS to generate reactive oxygen species (ROS). ${ }^{4}$ The PS absorbs photons of a certain wavelength and is thereby transformed into an excited state, which can dissipate its excitation energy by emission of heat or light, or by intersystem crossing to a triplet-state. There are two types of reactions that can take place once the PS is excited which can also occur simultaneously. The Type I pathway involves a hydrogen atom (or electron) transfer from the excited PS to a substrate such as a molecule or the cell membrane to form radicals, which in turn react with molecular oxygen species to produce ROS such as superoxide or hydroxyl radicals. In the Type II pathway, singlet oxygen is produced directly after the PS transfers energy to molecular oxygen. ${ }^{5}$ There are several different processes that could be initiated by PDT and are involved in tumour destruction. First, ROS can cause apoptosis or necrosis of tumour cells. In another mechanism, singlet oxygen can damage the vasculature of tumours. ${ }^{5}$ Finally, PDT can activate the immune response by membrane dysregulation of surface integrin- 
associated protein CD47 and damage-associated molecular patterns. $^{6}$

There are several factors that have an effect on PDT efficacy such as PS localization, the administration interval, the local oxygen concentration and the light exposure time. ${ }^{5}$ The localization of the PS is crucial due to the short half-life ( $<50 \mathrm{~ns})$ and radius of action $(<40 \mathrm{~nm})$ of singlet oxygen. ${ }^{7,8}$ As a consequence, sensitizers which are not internalized by cells usually show poor performance in PDT even if they produce large amounts of radicals. ${ }^{5}$ The wavelength and the type of light delivery also have a large effect on the efficacy of PDT. ${ }^{9}$ The applied wavelength influences not only the PS absorption efficiency, but also the penetration depth. Due to the wavelength-dependent scattering and absorption of skin, red light penetrates the skin and human tissues deeper than blue light, about $5 \mathrm{~mm}$ vs. $1 \mathrm{~mm}$, respectively. ${ }^{10}$

In this respect, two-photon excited photodynamic therapy (TPE-PDT) offers several benefits when compared to traditional one-photon excited PDT, such as deeper penetration of tissue. Due to the non-linear absorption, the PDT damage can be confined to a small excitation volume reducing collateral damage to the surrounding healthy tissue. In TPE-PDT, the PS is promoted to its excited state by absorbing two photons of a longer wavelength simultaneously during irradiation. Relaxation occurs via the Type I or Type II reaction with molecular oxygen as described previously. ${ }^{11}$ Due to the nonlinear behaviour of the absorption process, high peak power lasers such as modelocked Ti:sapphire lasers with femtosecond pulse durations are required to achieve efficient two-photon absorption. ${ }^{12}$ Different photosensitizers have been developed for TPE-PDT applications, including porphyrin derivatives, ruthenium complexes, conjugated polymers, and nanoparticles. ${ }^{11,13-23}$ The main considerations when choosing an optimal two-photon excitation photosensitizer (TPE-PS) are low dark toxicity, high two-photon absorption cross-section at a certain wavelength and a substantial triplet state quantum yield. ${ }^{24}$

Pre-clinical testing of TPE-PS presents some challenges. TPE-PDT has been tested in vivo using animal models to induce cancer regression in mice. ${ }^{25}$ The in vitro evaluation of treatment efficacy usually relies on 2D monolayer cell systems. However, there is increasing evidence that this setting does not provide sufficient comparison with in vivo conditions as the cellular microenvironment has a pronounced effect on the response to treatment. ${ }^{26,27}$ Microfluidic 3D cell cultures have been proposed previously to study the classical one-photon PDT efficacy but most of these systems rely on the qualitative assessment of cell viability using live-dead staining. ${ }^{28,29}$ However, an in vitro platform for quantifying the TPE-PDT efficiency using a hydrogel-based multicellular 3D cell culture has not been reported to date.

The aim of this study is to introduce a pre-screening platform of TPE-PS using a 3D osteosarcoma model together with adipose tissue-derived stem cells (ASC/TERT). Three different TPE-PS were used for testing the system, a two-photon photoinitiator P2CK, a fluorescent dye Eosin Y, and a porphyrin (TPP) (Fig. 1). The most extensively studied photosensitizers
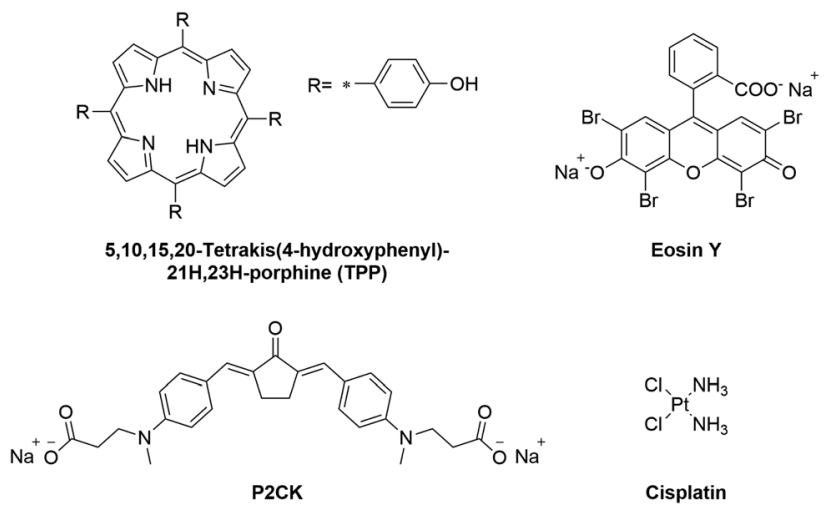

Cisplatin
$\mathrm{Cl}^{-} \mathrm{NH}_{3}$
$\mathrm{NH}_{3}$

Fig. 1 Molecular structures of the investigated two-photon photosensitizers (TPE-PS) and the reference compound cisplatin.

are in fact porphyrins, which contain four pyrrole rings with side chains with an absorption maximum usually centred around $400 \mathrm{~nm} \cdot{ }^{30}$ Eosin $\mathrm{Y}$ absorbs in the visible light region (490-650 nm) and has been proposed as a photosensitizer to inactivate bacterial biofilms using PDT. ${ }^{31}$ It has also been employed as a sensitizer for two-photon polymerization processes together with a co-initiator and an acrylate-based resin and for localized two-photon induced thiol-ene click reactions. $^{32,33}$ P2CK, a water soluble benzylidene cycloketonebased two-photon photoinitiator, has been used for twophoton polymerization of hydrogels. However, its use together with cells leads to poor cell survival and considerable phototoxicity in the concentration range $(>1 \mathrm{mM})$ required for the formation of stable hydrogel structures. ${ }^{34,35}$ On the other hand, when used as a two-photon sensitizer for cleavage of photolabile hydrogels, a much lower concentration is necessary $(<0.5 \mathrm{mM})$ that does not significantly harm cells outside of the focal volume. ${ }^{36}$ In order to optimize a working window for the proposed TPE-PS, the irradiation wavelengths should match the two-photon absorption maximum of the used PS. The $z$-scan technique, introduced in 1990, has become a standard method to characterize higher order nonlinearities such as two-photon absorption (2PA) cross-sections. ${ }^{37}$ Using a motorized stage, a thin sample (the sample length smaller than the Rayleigh length of the focused laser beam) is moved in and out of the focal plane of a laser beam along the $z$-axis. ${ }^{37,38}$ First, the 2PA cross-sections of the compounds in the 720-1000 nm spectral range were established using an inhouse developed automated $z$-scan setup. Afterwards, the cellular uptake of the TPE-PS was visualized using laser scanning confocal microscopy (LSM). The efficacy of the PS excited at their optimal wavelength was quantified via PrestoBlue metabolic assay using a 3D model of osteosarcoma cells encapsulated in a gelatin-based hydrogel. Finally, to show the precision of the irradiation, ASC/TERT cells, co-cultured together with osteosarcoma (MG63) spheroids, were visualized after selective two-photon irradiation of spheroids only in the presence of the PS using LSM. 


\section{Experimental}

All chemicals and cells were purchased from Sigma-Aldrich (Saint Louis, USA) unless otherwise stated. GraphPad Prism 6 was used for graphs.

\section{Spectral scanning of photosensitizers}

UV-VIS spectra were obtained using a PerkinElmer Lambda 750 spectrometer. Z-Scan measurements were performed on a home-built setup powered using a high power femtosecond laser oscillator (MaiTai DeepSee, Spectra Physics, Santa Clara, USA) with the tuning range of $690-1040 \mathrm{~nm}$, a repetition rate of $80 \mathrm{MHz}$ and a pulse duration of $70 \mathrm{fs} .{ }^{39}$ Using a motorized stage, the transmitted signal at 25 positions along the focused beam is recorded. A beam chopper with $90 \mathrm{~Hz}$ rotation frequency and an on-time of $78 \mu$ s reduces the exposure time of the sample to prevent thermo-optical effects caused by the high repetition rate of the laser. ${ }^{40}$ Each $z$-scan setting was measured in triplicate. Stock solutions of P2CK $(10 \mathrm{mM})$, Eosin $\mathrm{Y}(3 \mathrm{mM})$, and cisplatin $(1 \mathrm{mM})$ were prepared by dissolving the respective PS in PBS, while 5,10,15,20-tetrakis(4-hydroxyphenyl)-21H,23H-porphyrin (TPP) was dissolved in DMSO at a concentration of $10 \mathrm{mM}$. For each material, four different laser powers were used to verify that heat accumulation caused by the high repetition rate of the laser did not affect the measured 2PA behaviour.

\section{Two-photon irradiation setup}

The abovementioned laser was used throughout the TPE-PDT experiments with two different objectives (C-Achroplan 10×/ $0.3 \mathrm{NA}$, and $2.5 \times / 0.085 \mathrm{NA}$, ZEISS). ${ }^{34}$

The peak intensity ( $\left.I_{\text {peak }}\right)$ of the laser for a $\operatorname{sech}^{2}$ shaped pulse is calculated by the following eqn (1); ${ }^{41}$

$$
I_{\text {peak }}=\frac{\bar{P} \pi(\mathrm{NA})^{2}}{R \tau p \lambda^{2}}
$$

where $\bar{P}$ is the average laser power, NA is the numerical aperture, $R$ is the repetition rate, $\tau p$ is the pulse duration and $\lambda$ is the applied wavelength.

The full width at half maximum (FWHM) of the volumetric pixel (voxel) for the different objectives and wavelengths was calculated by the following eqn (2) and (3) and the results can be found in Table $1 ;^{42}$

$$
\begin{gathered}
\mathrm{FWHM}_{x, y}=\frac{0.32 \lambda}{\sqrt{2} \mathrm{NA}} 2 \sqrt{\ln 2} \\
\mathrm{FWHM}_{z}=\frac{0.532 \lambda}{\sqrt{2}}\left[\frac{1}{n-\sqrt{n^{2}-\mathrm{NA}^{2}}}\right] 2 \sqrt{\ln 2}
\end{gathered}
$$

where $\lambda$ is the applied wavelength, NA is the numerical aperture of the objective and $n$ is the refractive index of the material.

\section{Cell culture}

mCherry-labelled MG63 osteosarcoma cells and GFP-labelled adipose-derived stem cells (ASC/TERT1) (Evercyte, Vienna, Austria), provided by Ludwig-Boltzmann Institute for
Table 1 Full width at half maximum (FWHM) and the resulting volume of the voxel for the different objectives used in this study

\begin{tabular}{llllr}
\hline Objective & $\begin{array}{l}\text { Wavelength } \\
{[\mathrm{nm}]}\end{array}$ & $\begin{array}{l}\mathrm{FWHM}_{x, y} \\
{[\mu \mathrm{m}]}\end{array}$ & $\begin{array}{l}\mathrm{FWHM}_{z} \\
{[\mu \mathrm{m}]}\end{array}$ & $\begin{array}{l}\text { Volume } \\
{\left[\mu \mathrm{m}^{3}\right]}\end{array}$ \\
\hline 10×, 0.3 NA & 720 & 0.9 & 13.2 & 18.9 \\
Precision & 850 & 1 & 15.5 & 31.1 \\
& 960 & 1.2 & 17.5 & 44.8 \\
2.5×, 0.085 NA & 720 & 3.2 & 165.9 & 2672.6 \\
High-throughput & 850 & 3.8 & 195.8 & 4397.4 \\
& 960 & 4.3 & 221.2 & 6335.2
\end{tabular}

Experimental and Clinical Traumatology, were maintained at $37{ }^{\circ} \mathrm{C}$ and $5 \% \mathrm{CO}_{2}$ in an incubator. The transfection of the cells is described elsewhere. ${ }^{43}$ The osteosarcoma cells were cultivated in DMEM high glucose supplemented with 10\% FCS and $1 \%$ penicillin-streptomycin and the adipose tissuederived stem cells were cultured in EGM-2 (Lonza, Walkerville, MD, USA) supplemented with $10 \%$ FCS. The cells were detached using $0.5 \%$ trypsin-EDTA solution and centrifuged at 170 relative centrifugal force (RCF) for $5 \mathrm{~min}$. Afterwards, cells were re-suspended in the corresponding media and reseeded in T75 cell culture flasks (VWR, Radnor, PA, USA).

\section{Gelatin-norbornene synthesis}

Gelatin-norbornene with a degree of substitution (DS) of $90 \%$ was synthesized according to a previously reported protocol. ${ }^{44}$ In brief, for the preparation of $10 \mathrm{~g}$ of GelNB, $1.6 \mathrm{~g}$ of 5 -norbornene-2-carboxylic acid was dissolved in dry DMSO (Chem-Lab, Zedelgem, Belgium) under an inert atmosphere (Ar). After complete dissolution, $1.476 \mathrm{~g}$ (7.7 mmol) of 1-ethyl-3-(3-dimethylaminopropyl) carbodiimide (TCI Chemicals, Tokyo, Japan) was added to the reaction mixture together with $1.33 \mathrm{~g}$ (11.55 mmol) of $N$-hydroxysuccinimide followed by degassing 3 times. The mixture was allowed to react at room temperature for $25 \mathrm{~h}$ to eliminate any unreacted EDC functionalities. Next, $10 \mathrm{~g}$ of gelatin type B was dissolved in $150 \mathrm{~mL}$ of dry DMSO at $50{ }^{\circ} \mathrm{C}$ under $\mathrm{Ar}$ and reflux conditions. After complete dissolution, the prepared 5-norbornene-2-succinimidylester mixture was added to the gelatin solution followed by degassing 3 times. The reaction was allowed to proceed for $20 \mathrm{~h}$ followed by precipitating in a tenfold excess of acetone. Next, the precipitate was isolated using filtration (VWR, pore size 12-15 $\mu \mathrm{m}$, Radnor, USA) on a Büchner filter. After washing, the precipitate was re-dissolved in double distilled water $(\rho=$ 18.2 $\mathrm{M} \Omega \mathrm{cm}$ ) and dialysed (Spectra/por 4: MWCO 12-14 kDa) for $24 \mathrm{~h}$ at $40{ }^{\circ} \mathrm{C}$ against distilled water. After dialysis, the $\mathrm{pH}$ of the solution was adjusted to 7.4 using a $5 \mathrm{M} \mathrm{NaOH}$ solution, to obtain a clear solution. The gel-NB was isolated using freezing and lyophilisation. (Christ freeze-dryer Alpha 2-4 LSC, Martin Christ GmbH, Osterode am Harz, Germany).

\section{Cell viability assay}

$10 \mathrm{wt} \%$ gelatin-norbornene (GelNB, DS $=90 \%$ ) stock solution was prepared in PBS. MG63 cells were trypsinized and resus- 
pended in cell culture media ( 3 million cells per $\mathrm{mL}$ ) to achieve a final concentration of $5 \mathrm{wt} \%$ GelNB. Next, dithiothreitol in a $1: 1$ thiol-ene ratio $(10 \mathrm{mM})$ and $0.15 \mathrm{mM}$ lithium phenyl-2,4,6-trimethylbenzoylphosphinate (Li-TPO) were added. ${ }^{40}$ The optimal cell number for encapsulation was chosen to be 3 million per $\mathrm{mL}$ based on a preliminary experiment (Fig. S1†). $15 \mu \mathrm{L}$ of the hydrogel-cell suspension was added to each well of a glass bottom 384-well plate (Greiner Bio-One, Kremsmünster, Germany). Encapsulation was performed by 1 Joule UV-irradiation (368 nm) (Boekel UV Crosslinker, Boekel Industries, Feasterville, USA). ${ }^{44}$ In order to establish a working concentration for the PS, different concentrations of the PS were added and incubated for $4 \mathrm{~h}$. TPP was dissolved in DMSO at a concentration of $1 \mathrm{mg} \mathrm{mL} \mathrm{mL}^{-1}$ and diluted to the required concentration using cell culture media. The DMSO concentration never exceeded $0.1 \%$ of the media. Afterwards, the samples were washed and incubated for 2 days to remove any residual PS. The PrestoBlue metabolic assay was used according to the protocol provided by the manufacturer (Invitrogen, Carlsbad, USA). The emission at $590 \mathrm{~nm}$ was recorded at an excitation wavelength of $560 \mathrm{~nm}$ using a plate reader (BioTek Synergy H1, Winooski, USA). Afterwards, the efficacy of the light treatment was established. The highest non-toxic concentration of the PS was established to be $0.6 \mu \mathrm{M}$ for TPP, $0.5 \mathrm{mM}$ for P2CK and $0.25 \mathrm{mM}$ for Eosin Y. MG63 cells were encapsulated in the previously described manner and incubated with the PS for $4 \mathrm{~h}$ before they were two-photon irradiated at the respective optimal wavelength, $720 \mathrm{~nm}$ for TPP and $850 \mathrm{~nm}$ for P2CK using a $2.5 \times$ objective, with an applied peak intensity of $550 \mathrm{GW} \mathrm{cm}^{-2}$ and a laser writing speed of $4 \mathrm{~m} \mathrm{~s}^{-1}$. Afterwards, the wells were washed and the PrestoBlue assay was performed. Due to the low two-photon absorption of Eosin $\mathrm{Y}$ and the insufficient output of the laser system used at $960 \mathrm{~nm}$, Eosin Y was only used for cancer spheroid experiments.

\section{Cellular uptake of photosensitizers}

MG63 cells were seeded in a 96-well plate (5000 cells per well) and incubated overnight. The next day, solutions of the photosensitizers were added at concentrations of $0.5 \mathrm{mM}$ P2CK, $0.25 \mathrm{mM}$ Eosin $\mathrm{Y}$, and $0.6 \mu \mathrm{M}$ TPP. The cells were incubated for $4 \mathrm{~h}$ before laser scanning microscopy (LSM 700, Carl-Zeiss, Oberkochen, Germany) images were obtained using a $32 \times$ water-immersion objective (32×, 0.85 NA, ZEISS), using an excitation wavelength of $555 \mathrm{~nm}$ for P2CK and $488 \mathrm{~nm}$ for TPP and Eosin Y.

\section{Cell spheroid imaging}

In order to visualize that TPE-PDT can be restricted to the twophoton irradiated volume, MG63 spheroids co-cultured together with adipose-derived stem cells were used. Spheroids were produced using microtissue-molds according to a protocol described elsewhere. ${ }^{43}$ Once spheroids were formed after $24 \mathrm{~h}$, they were encapsulated together with 1 million per $\mathrm{mL}$ concentration of ASC/TERT-GFP cells in $5 \mathrm{wt} \%$ GelNB prepared following the abovementioned protocol. The cell containing hydrogels were incubated overnight and then imaged using LSM. Afterwards, different concentrations of the PS were added for $4 \mathrm{~h}$ and then spheroids were irradiated through a $10 \times$ objective using a peak intensity of $700 \mathrm{GW} \mathrm{cm}^{-2}$. After the PDT treatment, the hydrogel constructs were washed and then incubated for 2 days before LSM images were obtained. As a positive control, $30 \mu \mathrm{M}$ cisplatin was incubated with cells for $48 \mathrm{~h}$ before cells were imaged using LSM.

\section{Results and discussion}

In order to establish an optimal wavelength for TPE-PDT, the absorption spectrum for the different PS has to be determined. To this end, the two-photon absorption spectra were obtained by $z$-scan measurements. ${ }^{45}$ Since the linear UV/VIS absorption maximum of Eosin $\mathrm{Y}$ was at $506 \mathrm{~nm}$, the 2PA absorption region was estimated to be around $1000 \mathrm{~nm}$ (Fig. 2a). Although the $z$-scan assay did yield a change in the signal at the focus at $1000 \mathrm{~nm}$, this change was within the noise range of the setup and therefore did not allow the reliable extraction of a twophoton absorption cross-section $\left(\sigma_{2 \mathrm{PA}}\right)$. To overcome this limitation, the Eosin $\mathrm{Y}$ concentration was increased to $3 \mathrm{mM}$ for further measurements. These measurements indicated an optimal two-photon absorption window for Eosin $\mathrm{Y}$ at around 960-1000 nm. For cisplatin, a concentration of $1 \mathrm{mM}$ in PBS was sufficient to measure the nonlinear absorption. While both compounds exhibited nonlinear absorption behaviour, their 2PA were relatively low, i.e. below 100 Göppert-Mayer (GM, $1 \mathrm{GM}=10^{-50} \mathrm{~cm}^{4}$ s per photon per molecule). ${ }^{46,47}$ The $z$-scan analysis of a $10 \mathrm{mM}$ P2CK solution in PBS revealed an increase in $\sigma_{2 \mathrm{PA}}$ towards $700 \mathrm{~nm}$, and a local absorption maximum at $850 \mathrm{~nm}$ (405 GM).

$z$-Scan measurements of TPP were performed using a $10 \mathrm{mM}$ solution in DMSO. The results for TPP showed a 2PA cross-section of $112 \mathrm{GM}$ at $860 \mathrm{~nm}$ which decreased to $34 \mathrm{GM}$ at $980 \mathrm{~nm}$. At $1000 \mathrm{~nm}$, the signal dropped below the detection limit. The 2PA cross-section increased drastically towards $700 \mathrm{~nm}$ where the extracted 2PA cross-section was in the range of a several thousand GM (Fig. 2b). Due to this high $\sigma_{2 \mathrm{PA}}$, a concentration study was carried out (Fig. S2†). This was performed to test if a variation in concentration affects the 2PA behaviour. Therefore, three additional concentrations of TPP in DMSO $(0.5,1$ and $2 \mathrm{mM})$ were selected. Even at $0.5 \mathrm{mM}$, the signal drop at $700 \mathrm{~nm}$ was still $17 \%$, exhibiting significantly higher 2PA activity than the other screened substances, which is due to the larger conjugated $\pi$-system of TPP.

For the in vitro 3D TPE-PDT experiments, MG63 osteosarcoma cells were encapsulated in $5 \mathrm{wt} \%$ gelatin-norbornene (GelNB) hydrogel supplemented with a dithiothreitol (DTT) crosslinker in an equimolar thiol-ene ratio and the UV-photoinitiator Li-TPO $(0.15 \mathrm{mM})$ in a glass bottom 384 -well plate by UV-induced photo-polymerization. To establish the biocompatible concentration for each PS, Eosin Y and P2CK were dissolved in cell culture media. TPP was dissolved in DMSO at a concentration of $1 \mathrm{mg} \mathrm{mL} \mathrm{m}^{-1}$ and was further diluted in cell 
a)

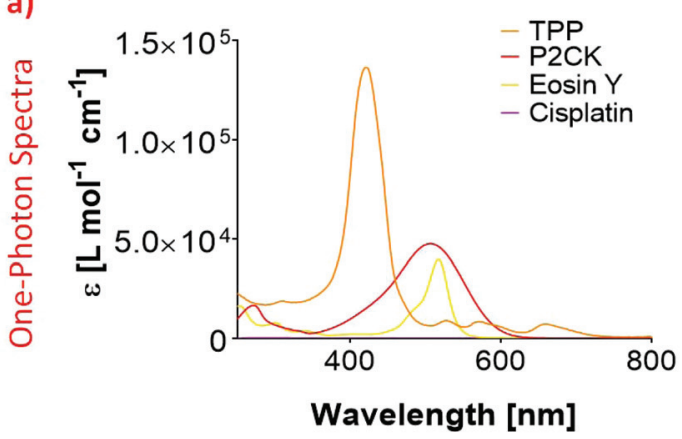

b)

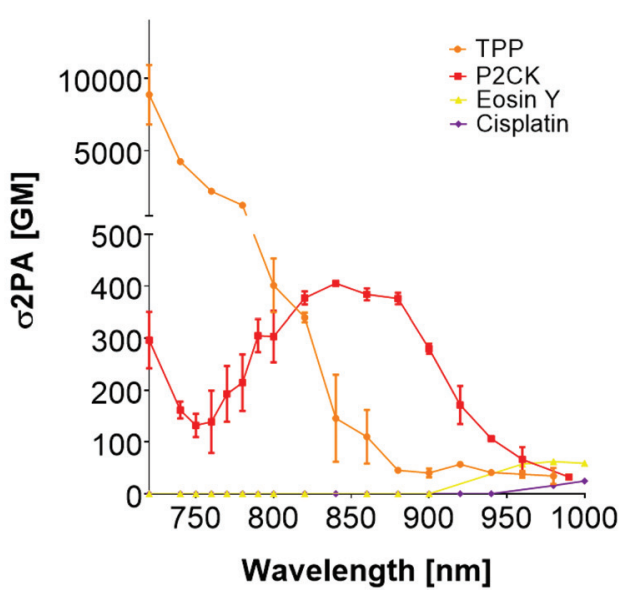

Fig. 2 One-photon and two-photon absorption spectra of the investigated compounds. (a) UV/VIS absorption spectra. The molar absorptivity coefficient $(\varepsilon)$ was calculated for the different substances. TPP showed the highest absorptivity with an absorption maximum at around $420 \mathrm{~nm}$

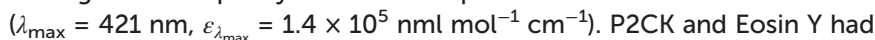

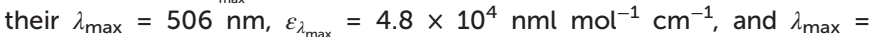

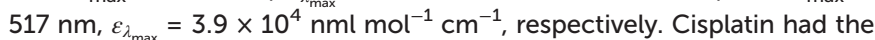

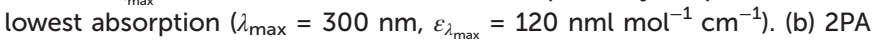
spectra of the photosensitizers. The two-photon absorption maximum of TPP can be found at $720 \mathrm{~nm}\left(\sigma_{2 \mathrm{PA}}=8865 \mathrm{GM}\right)$, that of P2CK at $850 \mathrm{~nm}\left(\sigma_{2 \mathrm{PA}}=405 \mathrm{GM}\right)$, Eosin $\mathrm{Y}$ has its maximum at $980 \mathrm{~nm}\left(\sigma_{2 \mathrm{PA}}=\right.$ $62 \mathrm{GM})$ and cisplatin has its highest $\sigma_{2 \mathrm{PA}}$ at $1000 \mathrm{~nm}\left(\sigma_{2 \mathrm{PA}}=25 \mathrm{GM}\right)$.

culture media, not to exceed $0.1 \%$ DMSO of the total volume. Different concentrations of the PS were added to the wells and incubated for $4 \mathrm{~h}$. The dark cytotoxicity of the PS was established by the PrestoBlue metabolic assay, which is based on a resazurin-based solution that is cell permeable and can be reduced inside the mitochondria of living cells. The fluorescent reaction product can be detected and quantified using a spectrometer/plate reader. The highest possible biocompatible concentration was used for the later experiments, highlighted in Fig. 3. P2CK was tolerated by the cells up to $0.5 \mathrm{mM}$, which was the highest among the substances, followed by Eosin Y with $0.25 \mathrm{mM}$ whereas TPP was non-toxic under dark conditions up to $0.6 \mu \mathrm{M}$.

Besides the 2PA spectra of the substances, which are vital to establish the optimal working window, the localization of the PS is also of crucial importance. Since most PS are fluorescent,

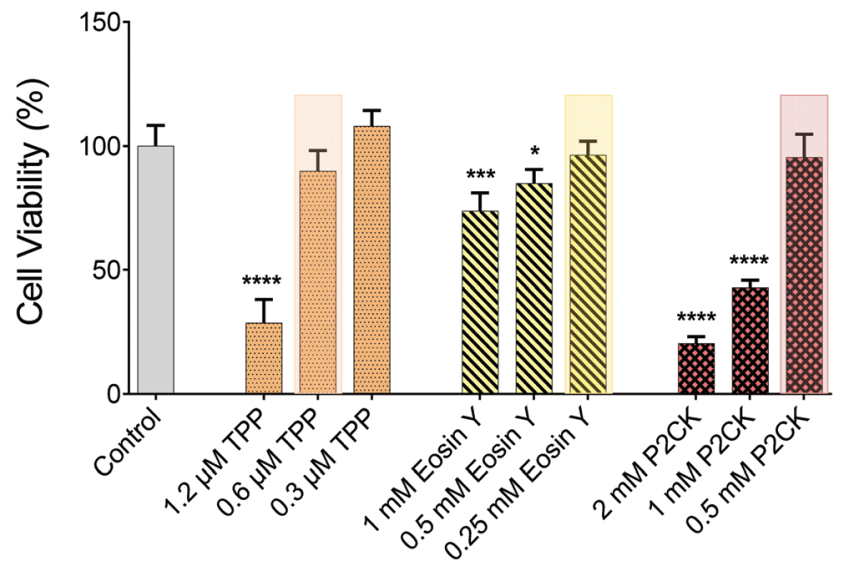

Fig. 3 Dark toxicity of the investigated PS. Encapsulated MG63 osteosarcoma cells were incubated with solutions of the PS in media for $4 \mathrm{~h}$ in the dark. Afterwards, the samples were washed and incubated for 2 days. The PrestoBlue metabolic assay was performed to quantify the viability of the cells. The highest acceptable concentration of the PS was then used for the PDT experiments, $0.3 \mu \mathrm{M}$ TPP, $0.25 \mathrm{mM}$ Eosin $\mathrm{Y}$ and $0.5 \mathrm{mM} \mathrm{P} 2 \mathrm{CK}$, respectively. The statistical significance was addressed by one-way ANOVA with Bonferroni post-test, with ${ }^{\star * * *} p<0.0001$, ${ }^{\star * *} p<0.001,{ }^{*} p<0.05(n=6)$.

a confocal laser scanning microscope (LSM) can be used to determine their accumulation. The PS was incubated with MG63 osteosarcoma cells using a 2D monolayer culture to monitor the cellular uptake. Eosin Y did not enter the cell membrane, while both TPP and P2CK can be traced within the cells (Fig. 4). These results suggest that Eosin Y might not work efficiently as a PDT agent. On the other hand, both P2CK and TPP show high potential for TPE-PDT based on their accumulation inside the cells in combination with their higher $\sigma_{2 \mathrm{PA}}$ at relevant wavelengths.

Once the biocompatible concentrations of the TPE-PS were established for dark conditions, MG63 cells, encapsulated in the GelNB hydrogel using the previously described procedure, were exposed to laser irradiation in the presence or absence of the TPE-PS. Each well was irradiated for 10 min using a peak intensity of $550 \mathrm{GW} \mathrm{cm}^{-1}$ at respective wavelengths. Due to the low output of the laser in the case of longer wavelengths
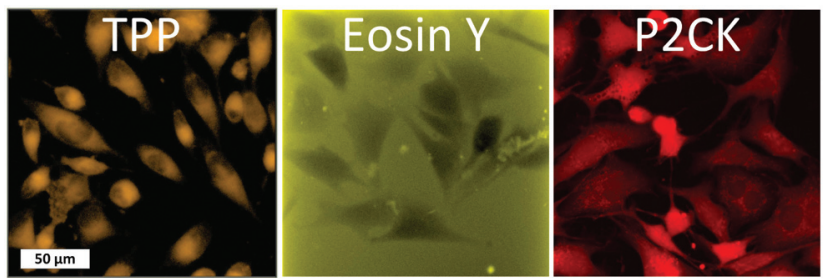

Fig. 4 Accumulation of PS in the cells. MG63 cells were incubated with the substances for $4 \mathrm{~h}$ prior to imaging with a confocal laser scanning microscope. TPP and P2CK both accumulated in the cells, while Eosin Y stayed mainly in the surrounding media. Concentrations of $0.5 \mathrm{mM}$ P2CK, $0.25 \mathrm{mM}$ Eosin $\mathrm{Y}$, and $0.6 \mu \mathrm{M}$ TPP were used for the localization experiments. 
(960 nm), only TPP and P2CK were used for quantitative assessment of the phototoxicity caused by irradiation. The wells were irradiated using a $2.5 \times$ objective with a NA of 0.085 . Therefore, the volumetric pixel (voxel) is expected to be over 100 -fold larger than in the case of the $10 \times$ objective, enabling a very high scanning speed of $4 \mathrm{~m} \mathrm{~s}^{-1}$ and the exposure of the whole well at the same time (Table 1). This permits the simple comparison of different compounds using the PrestoBlue assay, making the high throughput screening possible. Control wells which were only treated with $720 \mathrm{~nm}$ and $850 \mathrm{~nm}$ laser light in the absence of PS did not differ in cell viability compared to the non-irradiated controls, but both $0.5 \mathrm{mM}$ P2CK and $0.6 \mu \mathrm{M}$ TPP exhibited significant phototoxicity towards the cancer cells, leading to a viability of $27 \%$ and $15 \%$, respectively (Fig. 5). In the case of P2CK, this could be translated to a $65 \%$ and for TPP a 75\% decrease in cell viability upon irradiation at their optimal wavelengths when compared to the dark conditions.

One of the main advantages that TPE-PDT has to offer in comparison to conventional PDT is that the cellular damage can be reduced to the highly localized irradiated region, while conventional PDT can cause cell death in the surrounding tissue as well. The precision of the irradiation is dependent on the spot size of the laser, which is reliant on the numerical aperture of the objective. As a result, the irradiated volume can be kept relatively small when using a $10 \times$ objective with 0.3 NA (Table 1). As a proof of principle of the high spatiotemporal control, mCherry labelled MG63 spheroids (red) were encapsulated in a single cell suspension of GFP-labelled ASC/TERT cells (green). Only the spheroids were irradiated in the presence of the respective PS. As the cells are already fluorescently labelled, no additional live-dead staining was required to address the cell viability. If the cellular damage is caused by TPE-PDT, the damage will only appear in the irradiated regions, and if the interaction is mediated by one-photon

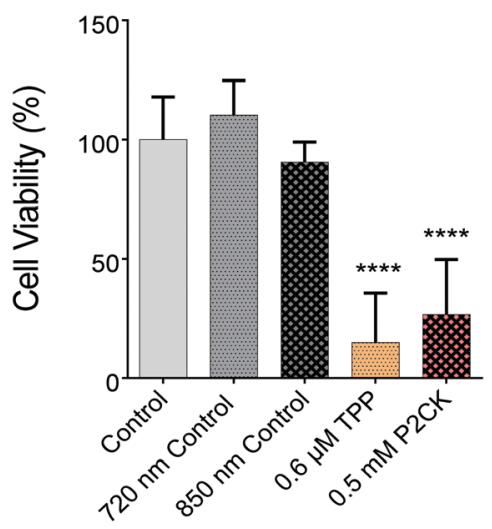

Fig. 5 Phototoxicity of PS. The control sample was not irradiated, while the TPP sample was irradiated with a $720 \mathrm{~nm}$ laser and the P2CK sample with an $850 \mathrm{~nm}$ laser with a peak intensity of $550 \mathrm{GW} \mathrm{cm}^{-2}$ and a scanning speed of $4 \mathrm{~m} \mathrm{~s}^{-1}$. Processing duration per well was $10 \mathrm{~min}$. The statistical significance was addressed by one-way ANOVA with Bonferroni post-test, with ${ }^{\star \star \star *} p<0.0001(n=6)$. absorption, the surrounding green cells will be impaired as well. The controls irradiated at $720 \mathrm{~nm}, 850 \mathrm{~nm}$ and $960 \mathrm{~nm}$ with a peak intensity of $700 \mathrm{GW} \mathrm{cm}^{-2}$ showed no negative effect on cell viability (Fig. S3†). $0.25 \mathrm{mM}$ Eosin Y irradiated at $960 \mathrm{~nm}$ also did not affect the cell viability. The green halo around the spheroids in the Eosin Y sample is probably a result from localized Eosin Y grafting into the hydrogel upon irradiation. The low efficiency of Eosin Y can be explained by its low cellular uptake (Fig. 4) and its low two-photon absorption in the spectral region accessible by the setup. Our study demonstrates that Eosin Y can be used in the presence of cells without significantly harming them. P2CK $(0.5 \mathrm{mM})$, on the other hand, causes substantial damage to the MG63 cell spheroid, both at $720 \mathrm{~nm}$ and $850 \mathrm{~nm}$, as it has a relatively high $\sigma_{2 \mathrm{PA}}$ at both these wavelengths (i.e. 292 GM and 405 GM respect-

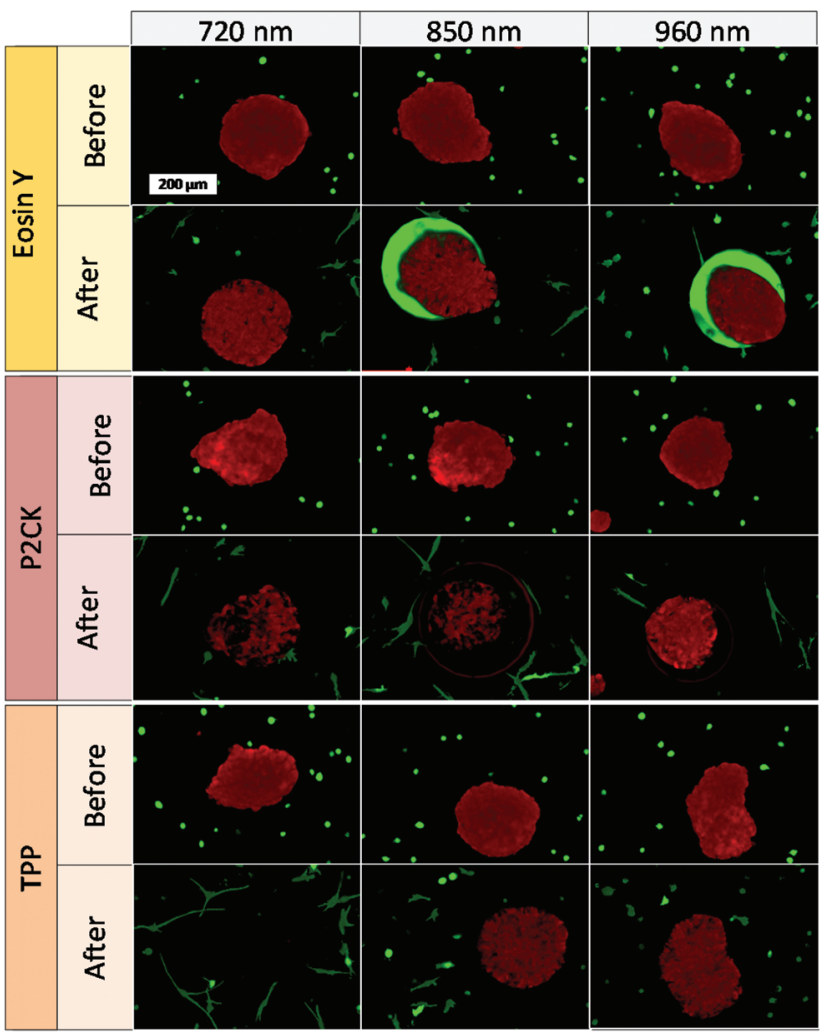

Fig. 6 TPE-PDT treatment of mCherry MG63 spheroids (red) surrounded with ASC-GFP single cells (green). The before images were taken right after the encapsulation of the cells. The irradiation of the samples was performed using different laser wavelengths. The region of irradiation was concentrated to the spheroids. One spheroid $(\varnothing 200 \mu \mathrm{m})$ was irradiated for $3 \mathrm{~min}$ using a peak intensity of $700 \mathrm{GW} \mathrm{cm} \mathrm{cm}^{2}$. The after photos were taken $48 \mathrm{~h}$ after treatment. Choosing the optimum wavelength of TPE-PS can increase the performance of the substances drastically. Eosin $Y$, even when it was irradiated at the optimal wavelength (960 nm and $58 \mathrm{GM}$ ), did not cause damage to cells. P2CK on the other hand caused damage both at $720 \mathrm{~nm}$ and $850 \mathrm{~nm}$ (296 GM and 405 GM, respectively). TPP eliminated the cancer cells completely when used at $720 \mathrm{~nm}$ (8865 GM) but not when it was irradiated at longer wavelengths, where its absorption is much lower (approximately $140 \mathrm{GM}$ at $850 \mathrm{~nm}$ ). 


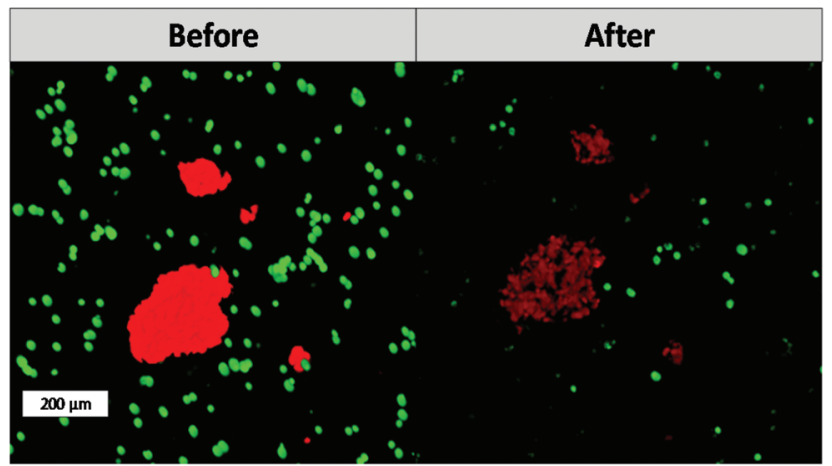

Fig. 7 Cell viability after treatment with $30 \mu \mathrm{M}$ cisplatin. Cisplatin affects both the viability of the ASC and MG63 cells.

ively). At $960 \mathrm{~nm}$, where its $\sigma_{2 \mathrm{PA}}(66 \mathrm{GM})$ is low, the spheroids remained intact. In the presence of TPP, MG63 spheroids were completely eliminated at $720 \mathrm{~nm}$ where TPP has a $\sigma_{2 \mathrm{PA}}$ as high as $8865 \mathrm{GM}$, while no significant damage was caused at higher wavelengths at which the $\sigma_{2 \mathrm{PA}}$ of TPP was below $100 \mathrm{GM}$ (Fig. 6). When compared to a common chemotherapeutic agent, cisplatin, the cell damage could be successfully restricted to the irradiated regions (Fig. 7), demonstrating the benefit of TPE-PDT in terms of localized targeted treatment.

\section{Conclusions}

In this study, we presented a systematic in vitro screening platform for TPE-PS. The automated $z$-scan setup enabled the determination of the two-photon absorption spectra of the investigated TPE-PS, providing indications about the optimal working window for each compound. Our results also demonstrated that the localization and the $\sigma_{2 \mathrm{PA}}$ of TPE-PS are of crucial importance for the PDT efficacy. Additionally, the TPE-PDT efficacy was quantified using a 3D model via the PrestoBlue metabolic assay, showing a significant reduction in cell viability after $2 \mathrm{P}$ irradiation in the presence of both P2CK and TPP. We were able to demonstrate the spatiotemporal control of TPE-PS using a 3D co-culture tumour model. The precision of TPE-PDT was evaluated by irradiating exclusively the osteosarcoma cells. In contrast to the use of cisplatin, no collateral damage to non-irradiated regions was observed. Our pre-screening approach enables high throughput profiling of TPE-PS, thereby allowing the comprehensive analysis of the PS efficacy in vitro.

\section{Conflicts of interest}

There are no conflicts to declare.

\section{Acknowledgements}

The authors thank Dr Severin Muehleder and Prof Wolfgang Holnthoner (Ludwig-Boltzmann-Institute for Experimental and Clinical Traumatology, Vienna, Austria) for providing the cells, and Prof. Liska (Institute of Applied Synthetic Chemistry, TU Wien) for providing P2CK. Funding from TU Biointerfaces Doctorate College is gratefully acknowledged. JVH holds an FWO-SB PhD grant (1S44016N) issued by the Research Foundation Flanders (FWO Vlaanderen).

\section{Notes and references}

1 Z. Zhang, W. Huang, M. Lei, Y. He, M. Yan, X. Zhang and C. Zhao, Int. J. Pharm., 2016, 498, 1-11.

2 S. Sharma, G. C. Brown and M. M. Brown, Ophthalmology, 6420, 2051-2059.

3 M. R. Hamblin and T. Hasan, Photochem. Photobiol. Sci., 2004, 3, 436.

4 D. E. Dolmans, D. Fukumura and R. K. Jain, Nat. Rev. Cancer, 2003, 3, 380-387.

5 T. J. Dougherty, C. J. Gomer, B. W. Henderson, G. Jori, D. Kessel, M. Korbelik, J. Moan and Q. Peng, J. Natl. Cancer Inst., 1998, 90, 40-45.

6 Y. Zheng, G. Yin, V. Le, A. Zhang, S. Chen, X. Liang and J. Liu, Int. J. Biol. Sci., 2016, 12, 120-132.

7 J. Moan, J. Photochem. Photobiol., B, 1990, 6, 343-347.

8 J. S. Dysart and M. S. Patterson, Phys. Med. Biol., 2005, 50, 2597-2616.

9 S. B. Brown, E. A. Brown and I. Walker, Lancet Oncol., 2004, 5, 497-508.

10 C. Ash, M. Dubec, K. Donne and T. Bashford, Lasers Med. Sci., 2017, 32, 1909-1918.

11 Z. Sun, L.-P. Zhang, F. Wu and Y. Zhao, Adv. Funct. Mater., 2017, 27, 1704079.

12 J. D. Bhawalkar, G. S. He and P. N. Prasad, Rep. Prog. Phys., 1996, 59, 1041-1070.

13 C. Fowley, N. Nomikou, A. P. McHale, B. McCaughan and J. F. Callan, Chem. Commun., 2013, 49, 8934-8936.

14 F. Bolze, S. Jenni, A. Sour and V. Heitz, Chem. Commun., 2017, 53, 12857-12877.

15 Y. Shen, A. J. Shuhendler, D. Ye, J.-J. Xu and H.-Y. Chen, Chem. Soc. Rev., 2016, 45, 6725-6741.

16 E. Wachter, D. K. Heidary, B. S. Howerton, S. Parkin and E. C. Glazer, Chem. Commun., 2012, 48, 9649-9651.

17 K. Ogawa and Y. Kobuke, BioMed Res. Int., 2013, 2013, 125658.

18 M. Lan, S. Zhao, Y. Xie, J. Zhao, L. Guo, G. Niu, Y. Li, H. Sun, H. Zhang, W. Liu, J. Zhang, P. Wang and W. Zhang, ACS Appl. Mater. Interfaces, 2017, 9, 14590-14595.

19 Y. Zhao, W. Wang, F. Wu, Y. Zhou, N. Huang, Y. Gu, Q. Zou and W. Yang, Org. Biomol. Chem., 2011, 9, 4168-4175.

20 J. Schmitt, V. Heitz, A. Sour, F. Bolze, H. Ftouni, J.-F. Nicoud, L. Flamigni and B. Ventura, Angew. Chem., Int. Ed., 2015, 54, 169-173.

21 H. A. Collins, M. Khurana, E. H. Moriyama, A. Mariampillai, E. Dahlstedt, M. Balaz, M. K. Kuimova, M. Drobizhev, V. X. D. Yang, D. Phillips, A. Rebane, 
B. C. Wilson and H. L. Anderson, Nat. Photonics, 2008, 2, 420-424.

22 M. Khurana, H. A. Collins, A. Karotki, H. L. Anderson, D. T. Cramb and B. C. Wilson, Photochem. Photobiol., 2007, 83, 1441-1448.

23 B. Gu, W. Wu, G. Xu, G. Feng, F. Yin, P. H. J. Chong, J. Qu, K.-T. Yong and B. Liu, Adv. Mater., 2017, 29, 1701076.

24 H. Abrahamse and M. R. Hamblin, Biochem. J., 2016, 473, 347-364.

25 J. R. Starkey, A. K. Rebane, M. A. Drobizhev, F. Meng, A. Gong, A. Elliott, K. McInnerney and C. W. Spangler, Clin. Cancer Res., 2008, 14, 6564-6573.

26 Y. Imamura, T. Mujohara, Y. Shimono, Y. Funakoshi, N. Chayahara, M. Toyoda, N. Kiyota, S. Takao, S. Kono, T. Nakatsura and H. Minami, Oncol. Rep., 2015, 33, 18371843.

27 A. G. Souza, I. B. B. Silva, E. Campos-Fernandez, L. S. Barcelos, J. B. Souza, K. Marangoni, L. R. Goulart and V. Alonso-Goulart, Curr. Pharm. Des., 2018, 24, 16891694.

28 Y. Yang, X. Yang, J. Zou, C. Jia, Y. Hu, H. Du and H. Wang, Lab Chip, 2015, 15, 735-744.

29 Y.-C. Chen, X. Lou, Z. Zhang, P. Ingram and E. Yoon, Sci. Rep., 2015, 5, 12175.

30 J. Moan, Photochem. Photobiol., 1986, 43, 681-690.

31 K. Marinic, D. Manoil, A. Filieri, J. C. Wataha, J. Schrenzel, N. Lange and S. Bouillaguet, Photodiagn. Photodyn. Ther., 2015, 12, 393-400.

32 M. Farsari, G. Filippidis, K. Sambani, T. S. Drakakis and C. Fotakis, J. Photochem. Photobiol., A, 2006, 181, 132-135.

33 C. A. Deforest and K. S. Anseth, Nat. Chem., 2011, 3, 925931.

34 M. Tromayer, A. Dobos, P. Gruber, A. Ajami, R. Dedic, A. Ovsianikov and R. Liska, Polym. Chem., 2018, 9, 31083117.
35 A. Ovsianikov, S. Mühleder, J. Torgersen, Z. Li, X. H. Qin, S. Van Vlierberghe, P. Dubruel, W. Holnthoner, H. Redl, R. Liska and J. Stampfl, Langmuir, 2014, 30, 3787-3794.

36 M. Lunzer, L. Shi, O. G. Andriotis, P. Gruber, M. Markovic, P. J. Thurner, D. Ossipov, R. Liska and A. Ovsianikov, Angew. Chem., 2018, 130, 15342-15347.

37 E. W. Van Stryland and M. Sheik-Bahae, Charact. Tech. Tabul. Org. Nonlinear Mater., 1998, 655-692.

38 M. Sheik-Bahae, A. A. Said, T.-H. Wei, D. J. Hagan and E. W. Van Stryland, IEEE J. Quantum Electron., 1990, 26, 760-769.

39 W. Steiger, P. Gruber, D. Theiner, A. Dobos, M. Lunzer, J. Van Hoorick, S. Van Vlierberghe, R. Liska and A. Ovsianikov, Fully automated z-scan setup based on a tunable fs-oscillator, 2019, submitted.

40 A. Gnoli, L. Razzari and M. Righini, Opt. Express, 2005, 13, 7976-7981.

41 B. Zhang, J. Zerubia and J.-C. Olivo-Marin, Appl. Opt., 2007, 46, 1819.

42 W. R. Zipfel, R. M. Williams and W. W. Webb, Nat. Biotechnol., 2003, 21, 1369-1377.

43 L. Knezevic, M. Schaupper, S. Mühleder, K. Schimek, T. Hasenberg, U. Marx, E. Priglinger, H. Redl and W. Holnthoner, Front. Bioeng. Biotechnol., 2017, 5, 25.

44 J. Van Hoorick, P. Gruber, M. Markovic, M. Rollot, G. Graulus, M. Vagenende, M. Tromayer, J. Van Erps, H. Thienpont, J. C. Martins, S. Baudis, A. Ovsianikov, P. Dubruel and S. Van Vlierberghe, Macromol. Rapid Commun., 2018, 1800181, 1-7.

45 A. Gnoli, L. Razzari and M. Righini, Opt. Express, 2005, 13, 7976-7981.

46 M. Göppert-Mayer, Ann. Phys., 1931, 401, 273-294.

47 W. Kaiser and C. G. B. Garrett, Phys. Rev. Lett., 1961, 7, 229-231. 